\title{
Clinical Analysis of Influenza A (H1N1) Viral Pneumonia Complicated with Bacterial Infection
}

\author{
Xixin Yan, Haibo Xu, Fangfang Qu, Yue Liu, Xiumin Zhang \\ Department of Respiratory Medicine, The Second Affiliated Hospital, Hebei Medical University, Shijiazhuang, China \\ Email: superxuhaibo123@163.com
}

Received November 13, 2012; revised December 24, 2012; accepted January 6, 2013

\begin{abstract}
Purpose: We investigated the efficacy of potent or combined antibiotics in patients suffering bacterial infections secondary to H1N1 by retrospectively analyzing their bacterial pathogen spectrum and clinical characteristics. Methods: Multi-center retrospective analysis was performed using clinical data of H1N1 patients from 27 hospitals in Hebei Province, China, from November 1 to December 31, 2009. Results: Of 480 H1N1-infected patients enrolled from an inpatient clinic, 91 were positive for bacterial culture. Bacteria were detected in sputum culture at $7.00 \pm 8.87$ days post-admission. Compared with the negative group, the patients in the positive sputum culture group had a higher mean age and prevalence of basic diseases, higher APECHEII (Acute Physiology and Chronic Health Evaluation II) score within 24 hours of admission, longer hospital stays, and higher mortality. In total, 189 bacterial strains were isolated, with the majority of samples testing positive for Acinetobacter baumanii (47), Streptococcus viridians (26), or Pseudomonas aeruginosa (19). S. viridians was the major cause of infection within 3 days of admission, while $A$. baumanii infection was more prevalent from 4 days post-admission; there was a significant difference in the constituent ratio between the two pathogens $(p<0.001)$. Compared with patients administered common antibiotics, the potent antibiotics group showed no significant difference in hospitalization time, time until bacterial detection, mortality, or detection ratio of resistant strains $(\mathrm{p}>0.05)$. Conclusions: Complicated bacterial infection in H1N1 patients increases hospitalization time and mortality. Gram-negative bacilli and multi-resistant strains are the main sources of infection. Early administration of potent or combined antibiotics, even during the period of rapid onset, may not be suitable in H1N1-infected patients, particularly previously healthy young patients.
\end{abstract}

Keywords: Influenza A H1N1; Bacteria; Antibiotics; Mortality

\section{Introduction}

The influenza A (H1N1) virus (2009) has universal susceptibility in population. Although there is no clear pattern of global incidence or data for patient mortality, there are three major causes of death in influenza A-infected patients: primary viral infection directly results in fatal respiratory failure; secondary bacterial infection affects the recovery of pulmonary function and ultimately results in death due to complications; and viral infection; or secondary bacterial infection induces deterioration of basic diseases. Previous investigations of the 1918-1919 influenza A pandemic found that most healthy young patients died from a single influenza viral infection, while mortality in older patients, particularly those with pre-existing conditions, was mainly due to complicated bacterial infection in the later stages of viral infection. From this standpoint, it is important to identify the number of cases of bacterial infection, and catalog the related clinical characteristics of influenza A (H1N1) patients, particularly where influenza A becomes a major infectious pathogen of the respiratory tract.

In this report, we described the bacterial pathogen spectrum and clinical characteristics of patients suffering bacterial infections secondary to $\mathrm{H} 1 \mathrm{~N} 1$, and analyzed the efficacy of potent or combined antibiotics.

\section{Materials and Methods}

\subsection{Patients}

The study included influenza A (H1N1) patients from 27 hospitals in Hebei Province, China, with samples collected from November-December 2009. The criteria for diagnosis of influenza A viral infection, severe and critical cases, met the Diagnosis and Therapy Strategy of Influenza A (H1N1) Virus (2009) requirements, as determined by the Ministry of Health of the People's Republic of China [1]. A confirmed case was a person whose influenza $A(p H 1 N 1)$ was verified by real-time reverse- 
transcriptase polymerase chain reaction (rRT-PCR) with or without the presentation of other clinical symptoms.

\subsection{Collection of Patient Data}

With the cooperation of the Department of Medical Policy, Provincial Health Bureau of Hebei, the data from original medical record were collected using a questionnaire that was designed according to the Clinical Research Project of Severe Influenza A (H1N1) Patients, established by the Division of Medical Policy, the Ministry of Health of the People's Republic of China.

\subsection{Statistical Analysis}

Means (standard deviations, SD) or medians (inter-quartiles, IQR) were calculated as summaries of continuous variables. For categorical variables, percentages of patients in each category were calculated. We compared clinical characteristics and clinical outcomes by using an ANOVA test, chi-square test, or Fisher's exact test or Wilcoxon rank-sum test as necessary. A $p$ value of less than 0.05 was considered to indicate statistical significance. All analysis was carried out using SPSS for Windows (release 13.0).

\section{Results}

\subsection{General Clinical Conditions}

In total, 480 influenza A (H1N1) patients were enrolled in Hebei Province, including 192 males and 288 females, with an average age of $30.1 \pm 18.7$ years, and hospitalization duration of 1 - 85 days (mean $12.9 \pm 10.4$ days). Sixty-one patients died during the course of the study $(12.7 \%)$, and the infection in 124 was further complicated by pre-existing conditions, including hypertension (47), diabetes (27), coronary artery disease (15), congestive heart failure (5), chronic obstructive pulmonary disease (10), stroke (5), hematological disease (10), and malignant tumors (5). Ninety-one patients showed positive bacterial culture (non-pre-existing), with an average hospitalization period of $7.00 \pm 8.87$ days. The clinical characteristics are listed in Table $\mathbf{1 .}$

\subsection{Pathogen Data}

A total of 189 bacterial isolates were identified from infected patients, including Acinetobacter baumannii (47), Streptococcus viridans (26), Pseudomonas aeruginosa (19), Staphylococcus aureus (16), Neisseria sp. (15), Klebsiella pneumonia (14), other Staphylococcus sp. (10), Stenotrophomonas maltophilia (8), and other species (34). There were 86 multi-drug resistant strains, including $S$. aureus (12), other strains (16), P. aeruginosa (5), and Acinetobacter calcoaceticus (4), as well as A. baumannii resistant to carbapenems (34), K. pneumonia producing extended-spectrum beta-lactamases (ESBL) (10), and Staphylococcus (5) resistant to methicillin.

The distribution of pathogens causing secondary bacterial infection and the clinical characteristics of patients within 3 days of hospitalization and at later time points are listed in Tables 2 and 3.

\subsection{Antibiotic Treatment}

There were 463 patients $(96.5 \%)$ treated with antibiotics during hospitalization. Review of the initial antibiotic strategy showed that 211 patients were administrated potent antibiotics (including carbapenems, $\beta$-lactams $/ \beta$ lactamase inhibitors against $P$. aeruginosa activity, fourth generation cephalosporins, vancomycin, teicoplanin, linezolid), while 200 patients were administrated a combination of two types of antibiotics. The prognoses of patients treated with the different antibiotic strategies for initial therapy post-admission are listed in Table 4.

\section{Discussion}

Previous influenza pandemics have shown that secondary bacterial infection and the aggression of underlying diseases are important factors contributing to mortality. Based on current data, influenza A (H1N1) patients with secondary bacterial infections are not uncommon. The

Table 1. Clinical characteristics of positive and negative bacterial culture groups.

\begin{tabular}{|c|c|c|c|}
\hline & Positive group & Negative group & $\mathrm{p}$ value \\
\hline Age (year) & $35.77 \pm 22.03$ & $28.74 \pm 17.59$ & 0.001 \\
\hline Hospitalization (days) & $20.23 \pm 14.35$ & $11.14 \pm 8.37$ & $<0.001$ \\
\hline Percentage of patients with basic disease & $29.0 \%$ & $17.7 \%$ & 0.04 \\
\hline Percentage of application of non-invasive ventilation & $12.09 \%$ & $13.88 \%$ & 0.65 \\
\hline Percentage of application of invasive ventilation & $54.94 \%$ & $14.14 \%$ & $<0.001$ \\
\hline Mortality & $20.88 \%$ & $10.80 \%$ & 0.009 \\
\hline
\end{tabular}


Table 2. Distribution of bacterial strains at different periods of hospitalization.

\begin{tabular}{ccc}
\hline & $\leq 3$ days & $>3$ days \\
\hline Acinetobacter baumannii & 1 & 46 \\
Pseudomonas aeruginosa & 2 & 17 \\
Streptococcus viridans & 13 & 13 \\
Staphylococcus aureus & 3 & 13 \\
Klebsiella pneumoniae & 2 & 12 \\
Staphylococcus & 0 & 10 \\
Neisseria & 6 & 9 \\
Otenotrophomonas maltophilia & 0 & 8 \\
Other & 4 & 30 \\
Total & 31 & 158 \\
\hline
\end{tabular}

There was a statistical difference in the constituent ratio of pathogens between within 3 days of hospitalization and at later time points group ( $p<$ $0.001)$.

Table 3. Clinical characteristics of patients with bacterial infection at different periods of hospitalization.

\begin{tabular}{cccc}
\hline & $\begin{array}{c}<3 \text { days } \\
(30 \text { cases })\end{array}$ & $\begin{array}{c}>3 \text { days } \\
(61 \text { cases })\end{array}$ & $\begin{array}{c}\mathrm{p} \\
\text { value }\end{array}$ \\
\hline Age (year) & $33.47 \pm 20.63$ & $36.48 \pm 22.60$ & 0.53 \\
WBC $(\times 109)$ & $7.05 \pm 4.64$ & $7.89 \pm 6.16$ & 0.50 \\
$\mathrm{~N}(\times 109)$ & $5.30 \pm 4.37$ & $6.29 \pm 5.43$ & 0.37 \\
$\mathrm{~T}\left({ }^{\circ} \mathrm{C}\right)$ & $37.31 \pm 1.09$ & $37.16 \pm 0.90$ & 0.49 \\
APECHEII score & $9.80 \pm 7.11$ & $9.80 \pm 5.64$ & 1 \\
$\begin{array}{c}\text { Hospitalization } \\
(\text { days })\end{array}$ & $14.72 \pm 7.71$ & $24.93 \pm 17.58$ & 0.003 \\
$\begin{array}{c}\text { Ratio of multi-drug } \\
\text { resistant bacteria }\end{array}$ & $25.81 \%$ & $49.37 \%$ & 0.003 \\
$\begin{array}{c}\text { Mortality } \\
\text { M. }\end{array}$ & $12.12 \%$ & $24.56 \%$ & 0.15 \\
\hline
\end{tabular}

Centers for Disease Control in the United States performed pathological analyses of 77 lethal cases of the most recent influenza A infection (2009) and found 22 cases with infection of the lower respiratory tract, including 10 cases with Streptococcus pneumoniae, 10 cases with other streptococcal infection, seven cases with $S$. aureus (five methicillin-resistant $S$. aureus), one case with Hemophilus influenzae, and 16 cases with underlying conditions such as asthma and cardio-cerebral vascular disease [2]. Researchers in Korea retrospectively analyzed reports from 115 deceased patients and found that 28 patients presented with positive bacterial sputum cultures, predominantly $S$. aureus and $K$. pneumoniae [3]. Louie et al. reported that in 1088 cases of influenza A (H1N1) infection, 46 cases were diagnosed with seconddary bacterial pneumonia, mainly caused by common pathogens such as $S$. pneumoniae, S. aureus, group A Streptococcus, and various Gram-negative bacteria. Most patients with secondary infection caused by Gram-negative bacilli suffered basic pulmonary diseases such as cystic fibrosis [4]. These results are similar to our present findings. Single-species infections caused by $S$. aureus, $S$. viridans, A. baumannii, and $P$. aeruginosa accounted for a certain percentage of disease, which is more common in the middle-aged and elderly populations already complicated with underlying conditions, and results in prolonged hospitalization and increased mortality. Differences in infectious isolates are related to region, climate, inclusion criteria, pathogen prevalence, drug administration, and drug resistance.

The bacteria detected in sputum cultures taken 3 days post-hospitalization were predominantly Gram-negative bacilli and multi-drug resistant bacteria. Of these, 54.9\% were detected in patients undergoing invasive ventilation, implicating these bacteria in ventilator-associated pneumonia. Interestingly, there was no obvious difference in the use of non-invasive ventilation between bacterial positive and negative groups, suggesting that there is no direct relationship between non-invasive ventilation and the detection of bacteria from the lower respiratory tract. This also indirectly implies that, in the therapy of influ-

Table 4. Comparison of prognoses between initial antibiotic strategies post-hospitalization.

\begin{tabular}{|c|c|c|c|c|}
\hline & $\begin{array}{l}\text { Potent antibiotics }{ }^{\mathrm{a}} \\
\quad(211 \text { cases })\end{array}$ & $\begin{array}{l}\text { Impotent antibiotics }{ }^{\mathrm{a}} \\
\quad(252 \text { cases })\end{array}$ & $\begin{array}{l}\text { Combined antibiotics }{ }^{\mathrm{b}} \\
\text { (200 cases) }\end{array}$ & $\begin{array}{c}\text { Non-combined antibiotics }{ }^{\mathrm{b}} \\
\text { (263 cases) }\end{array}$ \\
\hline Hospitalization time (days) & $12.75 \pm 9.74$ & $14.15 \pm 11.37$ & $12.86 \pm 9.28$ & $13.91 \pm 11.78$ \\
\hline Initial detection of bacteria (days) & $5.39 \pm 6.01$ & $8.05 \pm 10.08$ & $5.35 \pm 5.13$ & $8.78 \pm 11.20$ \\
\hline Mortality & $13.22 \%$ & $12.41 \%$ & $11.00 \%$ & $14.07 \%$ \\
\hline $\begin{array}{l}\text { Detection ratio of multi-drug } \\
\text { resistant bacteria }\end{array}$ & $35.48 \%$ & $50.00 \%$ & $46.51 \%$ & $43.48 \%$ \\
\hline
\end{tabular}

${ }^{\mathrm{a}}$ No statistical difference was observed between the potent antibiotic group and the Impotent antibiotic group in hospitalization time, initial detection of bacteria, mortality, or detection ratio of multi-drug resistant bacteria $(\mathrm{p}>0.05)$. ${ }^{\mathrm{b}}$ No statistical difference was observed between the combined antibiotic group and the non-combined antibiotic group in hospitalization time, initial detection of bacteria, mortality, or detection ratio of multi-drug resistant bacteria ( $\mathrm{p}>0.05$ ). 
enza A cases, non-invasive ventilation should be the first choice for respiratory support to correct hypoxemia, and should be performed as early as possible to avoid intubation $[5,6]$. These steps may reduce the risk of ventilator-associated pneumonia in the future.

Previous studies indicated that the incidence of secondary bacterial infection following influenza A (H1N1) infection tend to increase, which may be related to the facts that the virus can damage the mucosal epithelium of the airway, reduce the function of immune factors, and impair the bacterial defense of the organ [7,8]. Therefore, application of antibiotics is an important therapeutic strategy. However, antibiotics are not recommended for adult influenza patients who were previously healthy, are not complicated with pneumonia, or who have acute bronchitis without pneumonia. Previously healthy adult patients should only be given antibiotics if there are signs of deterioration, such as recurrent fever or obvious dyspnea. Generally, pneumonia in the middle and late stages ( $\geq 5$ days) is characterized by pulmonary consolidation with lobe or segment base as determined by imaging. Continuous fever and cough with yellowish purulent sputum suggest bacterial pneumonia and requires administration of antibiotics, which should be chosen as described above. Pneumonia acquired during hospitalizetion (including during a period of mechanical ventilation) for severe influenza should be treated with antibiotics appropriate for hospital acquired pneumonia [9]. Our results indicated that there were 31 cases of positive bacteria culture within 3 days of admission, mainly caused by Gram-positive cocci such as $S$. viridans, and the incidence of multi-drug resistant bacteria was $25.8 \%$. These etiological features suggest that antibiotics covering Gram-positive cocci should be the first choice for empiric treatment during the early stages of hospitalization. We found that administration of high-dose or combined antibiotics in the early stages could not shorten the hospitalization period, prevent bacterial infection, reduce the detection rate of multi-drug resistant bacteria, or decrease mortality, which may be related to the lower incidence of bacterial infection or multi-drug resistant bacterial infection of influenza A patients upon early hospitalization. Because the current influenza A virus represents a novel sub-type, the condition and prognosis of patients receives more social attention, and there is still no standard criteria for the detection of bacteria from the lower respiratory tract. Therefore, some of the detected bacteria may be commensal or even contaminating bacteria (non-quantitative culture), and excessive administration of highdose antibiotics may have serious consequences. Therefore, high-dose or combined antibiotics should not be the first choice of treatment for influenza A patients within 3 days of admission.

In summary, secondary bacterial infection following influenza $\mathrm{A}(\mathrm{H} 1 \mathrm{~N} 1)$ pneumonia is more common in the older population with complicated underlying conditions, which will prolong the duration of hospitalization and increase mortality. Nosocomial infection accounts for the majority of secondary infections, and influenza A patients should be closely monitored to detect any changes in the bacteria recovered from the lower respiratory tract. Secondary bacterial infection can be diagnosed early, by comprehensive analysis combined with sputum sampling and imaging of the lung. The empirical use of antibiotics and whether administration of antibiotics in the early stages of admission can improve long-term prognosis remain to be investigated. However, we conclude that it is not necessary to use high-dose or combined antibiotics as a first choice in the early stages of treatment.

\section{REFERENCES}

[1] The Ministry of Health of People's Republic of China, "Diagnosis and Therapy Strategy of Influenza A (H1N1) of the Ministry of Healthy of People's Republic of China (Version 3)," 2009. http://www.moh.gov.cn

[2] R. J. Leggiadro, "Bacterial Coinfections in Lung Tissue Specimens from Fatal Cases of 2009 Pandemic Influenza A (H1N1) - United States, May-August 2009," Morbidity and Mortality Weekly Report, Vol. 58, No. 38, 2009, pp. 1071-1074.

[3] H. S. Kim, J. H. Kim, S. Y. Shin, Y. A. Kang, H. G. Lee, J. S. Kim, J. K. Lee and B. Cho, "Fatal Cases of 2009 Pandemic Influenza A (H1N1) in Korea," Journal of Korean Medical Science, Vol. 26, No. 1, 2011, pp. 22-27. doi:10.3346/jkms.2011.26.1.22

[4] J. K. Louie, M. Acosta, K. Winter, C. Jean, S. Gavali, R. Schechter, D. Vugia, K. Harriman, B. Matyas, C. A. Glaser, et al., "Factors Associated with Death or Hospitalization Due to Pandemic 2009 Influenza A (H1N1) Infection in California," Journal of the American Medical Association, Vol. 302, No. 17, 2009, pp. 1896-1902. doi:10.1001/jama.2009.1583

[5] B.-W. Dai, W. Tan, L.-F. Sun, et al., "Clinical Analysis of 75 Severe and Critical Patients with Novel Influenza A (H1N1)," Chinese Journal of Practical Internal Medicine, Vol. 30, No. 1, 2010, pp. 6-9.

[6] T. Teke, R. Coskun, M. Sungur, et al., "2009 H1N1 Influenza and Experience in Three Critical Care Units," International Journal of Medical Sciences, Vol. 8, No. 3, 2011, pp. 270-277. doi:10.7150/ijms.8.270

[7] J. A. McCullers and K. C. Bartmess, "Role of Neuraminidase in Lethal Synergism between Influenza Virus and Streptococcus pneumoniae," The Journal of Infectious Diseases, Vol. 187, No. 6, 2003, pp. 1000-1009. doi: $10.1086 / 368163$

[8] J. A. McCullers, "Insights into the Interaction between Influenza Virus and Pneumococcus," Clinical Microbiology Reviews, Vol. 19, No. 3, 2006, pp. 571-582. doi:10.1128/CMR.00058-05

[9] N.-S. Zhong, C. Wang, G.-F. Wang, et al., "Guidance of Diagnosis and Therapy of Influenza," Journal of Community Medicine, Vol. 9, No. 5, 2011, pp. 66-74. 